\title{
The Media Functions of Network Sports Short Video in Communication
}

\author{
ZHANG Xueyan* \\ School of Media and Arts, Shanghai University of Sport, Shanghai, China \\ *Corresponding Author: ZHANG Xueyan, School of Media and Arts, Shanghai University of Sport, \\ Shanghai, China

\begin{abstract}
Sports and communication in modern society is not a separate existence and development, sports and communication are more and more closely linked. With the speed of the Internet and the rapid development of the Internet new media, sports communication presents many new features, among which the influence of short video on sports communication is more and more significant. Short video promotes sports communication more rapidly, and makes sports communication more purpose-oriented, popular, diversified, personalized and innovative. This paper makes an in-depth analysis of the functions and functions of short video in sports communication and points out that short video will play an increasingly important role in expanding the scope and influence of sports communication.
\end{abstract}

Key Words: Network new media, Short video, Sports communication, Medium

\section{INTRODUCTION}

With the rapid development and popularization of the Internet, it has become an important communication medium. With the development of mobile Internet and new media, as well as the popularity of smart phones, people can get and spread information without geographical and time restrictions. The speed of new media information transmission is faster and the means of transmission are more advanced. The emergence of short video, to some extent, has also had an important impact on people's lives. Short video plays an increasingly prominent role in various fields, especially in the field of sports communication.

Short video is a new thing emerging with the continuous development and improvement of network social media and the popularization of smart phones. At present, the research on short video is relatively few, and the research on the role of short video in sports communication is very limited. XUE Lan [1] explained that with the rapid development of modern network communication technology, China's new media and network media have incomparable advantages, and analyzed the status quo and development trend. ZHAO Yu et al. [2] analyzed the transmission status and characteristics of short videos, and illustrated the influence of short videos on the transmission of some audio-visual programs through some specific examples. DONG Qing et al.[3] took the World Cup in South Africa as an example to analyze the communication characteristics of media events in the era of new media. WANG Xiaodong et al. [4] studied the trend of media convergence and its significance to sports communication in the era of knowledge economy. ZHANG Lufeng [5] analyzed the hot trend of the application of short videos on domestic networks, and pointed out that the combination of short videos and news is a new way of news transmission.

Based on the latest development of short video, this paper focuses on the analysis of the functions and characteristics of short video in sports communication, and explores the role that short video should play in sports communication.

\section{FunCtions And FEATURES OF SHORT Video}

In recent years, with the development of social technology and network technology, new media has become a common term in daily life. Under the environment of new media, short video has gradually entered the life of the public. Different from live streaming and micro films, short video has low production threshold, short and simple production process, and people are more involved in the production of short video. Therefore, short video has greater dissemination value. 
Short video is short video, short and smooth features are extremely prominent. It is a high-frequency, fast-paced video broadcast on a variety of Internet new media platforms. The video duration is usually a few seconds, or a few minutes, usually within a minute. Short videos are closely connected with Internet communication, which is more suitable for audiences to watch in a state of mobile, or in a state of short time and leisure. Due to the short production cycle and interesting content of short videos, excellent short videos have a large number of stable and fixed fan groups. In recent years, "Weibo" and "Toutiao" have joined the short video industry, and the number of users of "MiaoHi", "MiaoPai", "Meituxiu", "Meipai", and "Weishi" of Tencent have shown a blowout growth trend [6-7].

\section{The Media Function Of SHORT Video In SPORTS COMMUNiCATION}

In recent years, short videos have played an increasingly important role in sports communication. Short videos are conducive to promoting sports communication more rapidly. Short sports videos correspond to the entertainment needs of people in the fast-paced life of modern society, and short sports videos are very consistent with the fragmented watching needs of young people [8]. Therefore, short video plays a very important role in sports communication in modern society.

\subsection{The Short Videos Have Helped Sports Spread More Quickly}

In the past, people know sports news mainly through TV and newspaper, but the audience can not always watch the game in front of the TV, can not always watch sports news in a fixed place. Now, the content of TV and newspaper can be presented in front of everyone through the network new media short video. People can learn a lot of sports information through the news in the short video, which undoubtedly speeds up the transmission speed of information.

At the same time, the mobile transmission mode of short video provides great convenience for the audience to watch the game and follow the news of the event, breaking the limitation of time and space. Short video is also characterized by fragmentation, which is conducive to enhancing the freedom of users to watch football. Short video can be applied in a variety of scenarios, such as buses, subways, high-speed trains, toilets and so on. In these places, people can use the fragmented time in their lives, so that they can obtain information anytime and anywhere, which is very convenient.

\subsection{The Short Video Promotes Sports Communication to Be More Purposeful, Diversified and Personalized}

Taking the World Cup as an example, the rise and development of short video and mobile live broadcasting make the forms of audience watching and participating in the World Cup more abundant and diversified. During World Cup, for example, fans in front of the TV watching at the same time, open your mobile terminals, in a mass of short video related to the World Cup to find their own interest, the content of the broadcast content, while the World Cup fans don't have a choice, but to see what kind of a short video about the World Cup, the audience themselves have the power, Fans can freely choose the content they want to watch in the World Cup. They can pay attention to the sports news and information they are interested in through sports short videos, with strong purpose and pertinence. Moreover, for the sports information they want to know, they will not miss the news of important sports events through the form of short videos [9-10].

\subsection{The Short Video to Promote the Focus of Sports Communication}

Generally speaking, a sports event is relatively long, but modern people's lifestyle is mostly fastpaced. Compared with the past, especially the life pace of young people in cities is faster, and it is difficult for the audience to concentrate on the game. More and more young people have lost the patience to watch a complete game. In its place is a fondness for highlights, tidbits and highlights, but the highlights of a full game are too scattered.

With the rapid development of the Internet, new media and smart phones, short video has become a bright spot in the field of sports communication. Due to the length of the short video, the wonderful part or wonderful moment will be concentrated together, focusing on the hot information, wonderful place. Therefore, if the audience chooses the fast-paced, frequent stimulation or highlights of sports short video to learn about sports news, the new way of sports communication, will focus on the highlights of a game and grasp the focus, so that the efficiency of watching the game will be higher. 


\subsection{The Short Video Has Increased the Sense of Audience Interaction and Participation in Sports Communication}

In the era of short video, in addition to watching games, fans can also have more interactions, such as thumb up, comments, forwarding and sharing, and more interactive ways. Short video can make the audience participate, which is also the charm of short sports video. With short videos, viewers can not only watch sports events, but also play them. Therefore, short video is conducive to improving the audience's interactivity and participation in sports events. It is precisely because of improving the audience's interaction and participation in sports events, so as to increase the audience to watch the game of fun. Therefore, short videos make sports communication more interesting and greatly enhance the audience's enthusiasm for watching sports events.

\subsection{The Short Video Makes Sports Communication More Entertaining and Popular}

The connection between sports and entertainment is extremely close. It is undeniable that sports play the role of leisure and entertainment, and short video itself has the function of entertainment. Therefore, short video is also widely used in sports and entertainment [11-12]..

The recording, broadcasting and live broadcasting of sports events on TV media generally require professional personnel to complete, but the production of short videos is not too high and relatively easy to operate. Therefore, short video makes sports dissemination more popular. At the same time, for ordinary users, especially for female users who are born without sports cells, this mode of simplifying the complex is conducive to making sports communication more popular, because in many cases, the simple public expression is more marketable than the professional expression, which is conducive to gaining the popularity and pursuit of a wide audience.

\subsection{The Short Videos Increase the Diversity and Cultural Influence of Sports Communication}

For China, in the past, most of the audience received sports news through sports newspapers or TV, but the transmission form of new media like short video has made the channels of sports transmission more novel and more diversified. For example, in the dissemination of sports news, short video can also provide viewers with various functions such as playback. When the audience misses a certain event or a wonderful moment of a certain event, the audience can make up directly from the short video of sports, and then fully feel the charm of sports competition.

With the emergence of short video, it provides a new way for sports communication. For sports communication, it further expands the scope and influence of sports communication. With the popularity of smart phones and the increase of mobile phone users, the dissemination of sports news through short videos to a large extent expands the scope of the audience, and the expansion of the audience scope is of great help to improve the cultural influence of sports news.

\section{CONCLuSion}

With the increasingly mature development of mobile Internet in China, the widespread popularity of smart phones and the gradual diversification of users' social demands, short video emerges as The Times require. Short video also sets off a new round of development boom in China, and its popularity is based on the new media environment and new user needs. Although the short video in China has not been produced for a long time, short video and sports communication are closely combined and closely related. Short video will definitely play a more and more important role in sports communication.

\section{REFERENCES}

[1] XUE Lan. Research on the Current Situation, Trend and Development of Network Media on Sports Information Communication -- Taking the World Cup Soccer as an Example. Physical time and space, 2012 (9), 130-131.

[2] ZHAO Yu, WANG Yongze, MA Xin. Short video transmission of the status quo analysis. Broadcasting and television information, 2015 (9), 53-55.

[3] DONG Qin, HONG Yan, CHEN Jie. Analysis of the new media era sports communication. Sport Culture Guide, 2011 (7), 146-149.

[4] WANG Xiaodong, Li Zonghao. On the change of media form and its influence on sports communication. Journal of Shanghai Sport University, 2003 (5), 29-31. 
[5] ZHANG Lufeng. A short video as news spreading prospects of the development of new ways. News Knowledge, 2016 (7), 38-40.

[6] DUAN Jingyu. Based on the causes of formation of hot sports short video analysis and value . News Front, $2018(2), 120-121$.

[7] ZHOU Jinyu, WANG Xiangfei, CUI Qiyao. Large sports short video transmission study. Sport Culture Guide, 2018 (7), 154-158.

[8] LUO Xuan. Situation and motivation of the new media sports communication. Young Reporters, 2015 (27), 72-73.

[9] LI Fang, WEI Chaoyang. Mobile news client large sports events communication difficulties and development strategy study . Journal of Shenyang sport University, 2015 (5), 80-86.

[10] MAO Ruizhe. Research on the characteristics and trends of sports news APP communication: A case study of Tencent sports APP. Young Reporter, 2016 (26), 87-88.

[11] YAN XiaoFang. The spread characteristic of the mobile short video and media opportunities. Southeast Communication, 2016 (2), 90-92.

[12] ZHANG Yushu. "To read" short video from the current situation of the development of media and cultural interpretation. New Media Research, 2017 (5), 123-124.

\section{AUTHOR'S BIOGRAPHY}

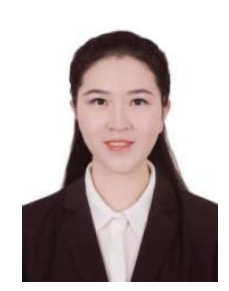

ZHANG Xueyan, Master of Fine Arts(MFA), School of Media and Arts, Shanghai University of Sport, Shanghai, China. The main research direction is research on modern media art. Email: dchzhang2007@163.com

Citation: ZHANG Xueyan. "The Media Functions of Network Sports Short Video in Communication" International Journal of Humanities Social Sciences and Education (IJHSSE), vol 8, no. 6, 2021, pp. 61-64. doi: https://doi.org/10.20431/2349-0381.0806009.

Copyright: (C) 2021 Authors. This is an open-access article distributed under the terms of the Creative Commons Attribution License, which permits unrestricted use, distribution, and reproduction in any medium, provided the original author and source are credited. 